\title{
Derivation of Explicit Solutions Describing Early Stages of Platelet Activation
}

\author{
Rachel Austin, Scott Fones*, Dominic Santoleri, Kaitlyn Thomesen, Pak-Wing Fok \\ Department of Mathematical Sciences, University of Delaware, Nerwark, DE \\ Students: rraustin@udel.edu,*sfones@udel.edu,dsantole@udel.edu, thomesen@udel.edu \\ Mentor: pakwing@udel.edu
}

\begin{abstract}
The formation of blood clots is vital for biological repair of injured blood vessels. When a blood vessel is injured, platelets come into contact with collagen, causing glycoprotein VI (GPVI) to undergo a conformational change and initialize the clotting process. This project aimed to simplify and solve a system of coupled ordinary differential equations (ODEs) proposed in Model A of Regulation of Early Steps of GPVI Signal Transduction by Phosphatase: A Systems Biology Approach by JL Dunster et al., modeling early platelet activation kinetics. In doing so, the ODEs were non-dimensionalized and the approximate analytical solutions were then found. The approximate solutions compare favorably to the numerical solutions and provide deeper insight into the signal regulation pathway. Most notably, the solutions expose a time at which the pathway dynamics change drastically. This illustrates the critical role of cytosolic spleen tyrosine kinase (Syk) as a molecular timer in the cascade.
\end{abstract}

\section{KEYWORDS}

Platelet Activation; Signal Cascade; Coupled Differential Equations; Asymptotic Analysis; Systems Biology; Mathematical Modeling; Model Simplification; Glycoprotein VI; Spleen Tyrosine Kinase; Syk Activation Dynamics

\section{INTRODUCTION}

Blood is composed mostly of plasma, white blood cells, red blood cells, and platelets. Platelets are anuclear, biconcave discs responsible for the detection and repair of structural damage to the blood vessel wall. The extracellular matrix, ECM, surrounding blood vessels is composed mainly of collagen. When the blood vessel wall is compromised, the platelets inside the blood vessels are exposed to the ECM (Figure 1a). The platelets respond to the presence of collagen by initiating a repair pathway. ${ }^{1}$ More specifically, the interactions with glycoprotein VI (GPVI), cause the platelet to undergo a conformational change that initiates the clotting process (Figure 1b). This conformational change starts a signaling cascade that relies heavily on the phosphorylation of secondary messengers to amplify the signal. This helps to aggregate additional platelets to the wound, and allows clot formation to occur. Once the clot begins to form, regulatory feedback loops within this signaling cascade modulate the strength and duration of the signal. Although the forward signaling cascade has been well studied, little information exists on regulation of the signal. Evidence indicates that as the signaling process occurs, products further downstream interact with earlier events to regulate and modify the strength and duration of the clotting response. ${ }^{1}$

The inappropriate activation of clot formation can lead to thrombosis, heart disease, and stroke, all of which are potentially fatal. ${ }^{3}$ Developing a model to depict this signaling pathway can lead to a better quantitative understanding of clotting. Through mathematical modeling, physicians can better detect and target crucial reactions in this pathway. These models could potentially aide in the development of medication that prevents inappropriate clot formation, making treatment more effective. In addition, spleen tyrosine kinase, Syk, plays a significant role in immune cell signaling. ${ }^{4}$ This role may be better understood though modeling Syk's dynamics in the context of platelet activation. 
Three models for the initial steps of the signal cascade for platelet activation were developed by Dunster et al. ${ }^{1}$ Their simplest model, Model A, proposes seven differential equations correlating to the different steps and participants in the cascade. These equations are useful in developing a model, but the information obtained from a system of differential equations is limited. Finding analytic approximations for these equations can provide more insight into the model, such as the concentration of each component of the cascade at a given point in time.

After highlighting the importance and current understanding of this pathway in section 3, we introduce a model proposed by Dunster et al. in section 4.1. In section 5.1, these equations are non-dimensionalized to obtain unitless constants that can be compared directly. We proceeded to simplify and solve the seven differential equations to obtain solutions in section 5.6. In section 6, we offer conclusions and insights provided by the analytical solutions.

\section{Literature Review}

Dunster et al. have used their understanding of the clotting cascade pathway to develop a model of the signaling pathway. ${ }^{1}$ However, the authors concede that the negative feedback loops are not well understood. Other mathematical models for platelet signaling tend to focus on signal activation and have been remarkably successful in predicting calcium regulation in platelets during signal transduction. ${ }^{5}$ These models typically focus on forward signal transduction while signal regulation mechanisms remain largely unstudied. These feedback inhibition pathways are crucial for understanding the regulation of this pathway and the steady state level for phosphorylated spleen tyrosine kinase, Syk. Several potential feedback processes are considered and incorporated in later, more complex modeling systems by Dunster et al. ${ }^{1}$ Such a feedback loop was investigated, with particuar emphasis placed on the question of whether the ligand T-cell ubiquitin ligand-2, TULA-2, is partially responsible for the regulation of phosphorylated Syk. ${ }^{6}$ A negative correlation between the concentration of TULA2 and activated Syk levels was discovered, in addition to an apparent specificity of TULA-2 for Syk. Not only is TULA-2 suspected to negatively regulate activated Syk, but c-Cbl, a kinase from the Src family that is activated along the clotting signal cascade may also be involved. Studies have also indicated a potential regulatory role that c-Cbl has on activated Syk. ${ }^{7}$ These feedback loops are incorporated into the models from the Dunster et al. paper to predict activated Syk concentrations. The incorporation of these loops is further supported by the key role Src family kinases play in initiating platelet activation. ${ }^{8}$ The regulatory role of phosphatases can be considered in modulating the cellular response to signaling. ${ }^{9}$ Phosphatases also play a regulatory role in the activation of Syk and the maintenance of the clotting signal.

To further support the many checkpoints and feedback loops in this physiological phenomenon, the idea of Syk being the main checkpoint for this cascade has been studied. ${ }^{10}$ Knowing that Syk activation is heavily regulated supports the assumption that Dunster made when focusing on the concentration of activated Syk as an indicator of the clotting signal progression. The Y525 motif on Syk, which is the location where a tyrosine residue becomes phosphorylated during Syk activation, is important for regulation and activation. ${ }^{10}$ ITAM-Syk binding and later Syk activation heavily relies on this motif. Most of the feedback mechanisms, such as phosphatase dephosphorylation of Syk, depend on protein recognition of Y525.

The mechanisms behind the GPVI activation are also vital to the understanding of the clotting signaling cascade. ${ }^{2}$ Dunster et al.'s mathematical model of platelet activation uses this to explain the high affinity of Syk for ITAM on the GPVI receptor, leading to Syk strongly binding to the phosphorylated receptor complex. Additional research explores platelet activation and blood coagulation as complementary processes, illustrating the importance of regulatory processes in ensuring efficient responses to injury. ${ }^{11}$

Understanding the complex mechanisms of platelet activation allows for more effective antithrombotic medicines targeting the prevention of errant clot formation. Arterial thrombosis is an acute complication that forms on chronic lesions of atherosclerosis and results in heart attacks and stroke. ${ }^{3}$ Further exploration of platelet activation will lead to a better understanding of the formation and progression of atherosclerotic plaque. As the most common cause of mortality in developed countries, there are large clinical implications to a deeper understanding of clotting dynamics. ${ }^{1,3}$ Beyond atherosclerosis, 
platelet activation contributes to the long-term recovery outcomes of heart transplantation. ${ }^{12}$ Long-term survivors of heart transplantation show increased activation of platelets, potentially contributing to increased immune activation and complications. ${ }^{3}$ As a component of endothelial dysfunction, the inverse relationship between platelet activation and endotheliumdependent vasomotor function appears to explain the connection between vasomotor dysfunction and atherothrombotic events. ${ }^{13}$ These phenomena, along with other connections between platelet activation and chronic illness, illustrate the importance of understanding the role of platelet activation.

\section{GOVERNING EQUATIONS}

Description of Dunster's Model A

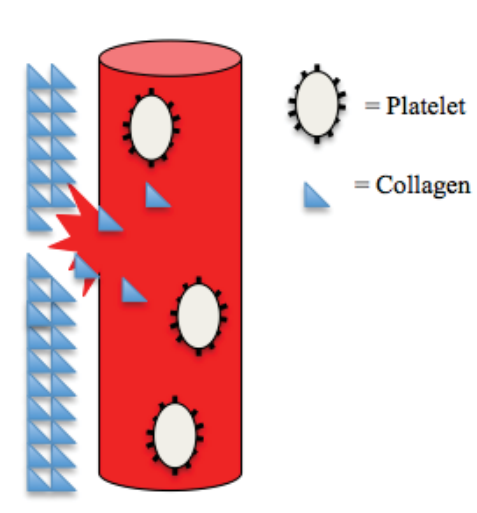

(a)

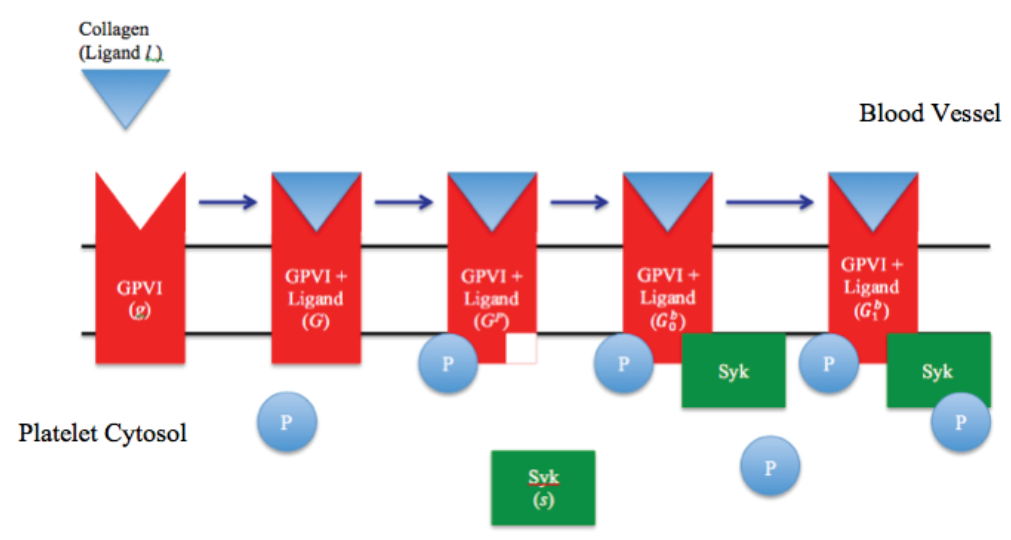

(b)

Figure 1. (a) Damage to the blood vessel wall exposes platelets to collagen. Other blood vessel components (red blood cells, white blood cells, etc. have been excluded for simplicity. (b) Exposure of GPVI at the platelet surface to collagen begins the platelet activation cascade by forming a receptor complex. The receptor complex is then phosphorylated and Syk binds to the complex, activating the receptor and initiating downstream signaling.

This paper analyzes the intermediates in Model A of the platelet activation signaling pathway developed by Dunster et al. ${ }^{1}$ Collagen normally exists outside of the blood vessel, but upon damage to the blood vessel, collagen gets exposed to the bloodstream and platelets (Figure 1a). Collagen or the multivalent GPVI-selective agonist, collagen-related peptide (CRP), used during the experiments (both denoted as $l$ ) exposed to the platelets in the extracellular matrix can then interact with GPVI at the cell surface. As the ligand reversibly binds to the free GPVI receptor, $g$, on the cell membrane surface, the receptor-ligand complex, $G$, forms. The reverse reaction then involves the dissociation of $G$ back into $l$ and $g$. Following the formation of the receptor-ligand complex, it becomes phosphorylated, $G^{p}$. This phosphorylation allows for recruitment and activation of the cytosolic protein-tyrosine kinase, Syk, found inside the platelet cytosol. Syk, denoted $s$, binds to the phosphorylated receptor complex, $G^{p}$, to form $G_{0}^{b}$. Since GPVI associates with the ITAMs, when Syk aggregates and interacts with the activated ITAM, its tyrosine 525 residue (Y525) gets phosphorylated $\left(G_{1}^{b}\right)$. This entire process, resulting in the phosphorylation of Syk, initiates the downstream signaling. The high binding affinity of Syk to the ITAM occurs through two bound tyrosine residues, resulting in protection of the ITAM from dephosphorylation. The receptor-ligand complex formation and the phosphorylation of Syk steps are reversible and have the potential for being regulated by outside enzyme or downstream products. Figure $1 \mathrm{~b}$ demonstrates the mechanism described above, and Table 1 and Table 2 detail the variables and parameters involved for this cascade along with the values for each, respectively.

The rate at which each reactant, intermediate, and product in the mechanism shown in Figure $1 \mathbf{b}$ is created or consumed can be modeled by the seven differential equations determined by Dunster et al. (Equations (1a-1g)). ${ }^{1}$ Equations $1 \mathrm{a}$ and $1 \mathbf{b}$ are very similar and measure the rates of ligand and GPVI consumption $\left(\frac{d l}{d t}=-\frac{k_{1}}{V_{e} A_{v}} g l+\frac{k_{-1}}{V_{e} A_{v}} G\right.$, for $l$ and $\frac{d g}{d t}=-k_{1} g l+k_{-1} G$ for $g$ ). The first term in these equation demonstrates the consumption of $l$ and $g$ through their association to create $G$ while the second term represents the reverse reaction where $G$ dissociates back into $l$ and $g$. Because $g$ is fixed to the platelet 
membrane, it can only interact with $l$ when it is close to the membrane. As such, the ligand rate equation, Equation 1a, is scaled down by the volume of the extracellular matrix $\left(V_{e}\right)$ so that only the ligand molecules close to the platelet membrane are accounted for in the equation. Additionally, Equation 1a is divided by Avogadro's Number $\left(A_{v}\right)$ to convert the mM units $\left(\frac{\text { moles }}{\mathrm{m}^{3}}\right)$ of $l$ to molecules like $g$.

The first two terms of Equation 1c are the opposite of those in Equations 1a and 1b. These terms represent the same forward and reverse reaction involving ligand-receptor binding, but presented from the alternate view. The direction that consumes $l$ and $g$ creates $G$ and the direction that creates $l$ and $g$ consumes $G$, hence the opposite signs. The final term in Equation 1c accounts for the rate that $G$ is consumed in the next step of the pathway as it gets phosphorylated to $G^{p}$. Together, these terms outline how quickly $G$ is created at the beginning of the reaction and then consumed in both forward and reverse reactions $\left(\frac{d G}{d t}=k_{1} g l-k_{-1} G-k_{2} G\right)$.

$G^{p}$ creation depends on $G$ consumption. Hence the first term of Equation $1 \mathrm{~d}\left(\frac{d G^{p}}{d t}=k_{2} G-\frac{k_{3}}{V_{p} A_{v}} G^{p} s\right)$ is the opposite of the last term of Equation 1c. The dephosphorylation of $G$ is not significantly reversible and so there is no term denoting the reverse of this reaction. This is due to the speed with which the next step of the pathway occurs. $G^{p}$ interacts with $s$ from the cytosol to create $G_{0}^{b}$. This reaction creates the second term in Equation 1d and the rate equation for $s$, Equation 1e $\left(\frac{d s}{d t}=-\frac{k_{3}}{V_{p} A_{v}} G^{p} s\right)$ which only participates in this reaction within the pathway. Because $s$ exists in the cytosol similarly to how $l$ exists in the extracellular matrix, this term has to be adjusted to account for the fact that this interaction can only occur along the membrane. However, since $s$ is cytosolic instead of extracellular, the volume of the platelet $\left(V_{p}\right)$ is used in the calculation instead of the external environment. Additionally, the rate constant for this step of the reaction $\left(k_{3}\right)$ is so large that the reaction drives forward, consuming $G^{p}$ as quickly as it is created.

The last two equations represent the final part of this pathway. $G_{0}^{b}$, representing the ligand-bound receptor interacting with Syk, gets phosphorylated to create $G_{1}^{b}$ which can further initiate downstream signaling. The first term of Equation 1f $\left(\frac{d G_{0}^{b}}{d t}=\frac{k_{3}}{V_{p} A_{v}} G^{p} s-\rho_{1} G_{0}^{b}+\gamma_{1} G_{1}^{b}\right)$ shows the irreversible creation of $G_{0}^{b}$ while the last two terms show the forward and reverse rates of its reversible phosphorylation into $G_{1}^{b}$. Similarly, Equation $\lg \left(\frac{d G_{1}^{b}}{d t}=\rho_{1} G_{0}^{b}-\gamma_{1} G_{1}^{b}\right)$ shows the same forward and reverse reactions but in the opposite direction. As such, these two protein forms eventually reach an equilibrium once all of $s$ is consumed by the previous reaction.

\begin{tabular}{|c|c|c|c|}
\hline Variable & Description & Units & Initial Value \\
\hline$l$ & Ligand & $\frac{\text { moles }}{\mathrm{m}^{3}}(\mathrm{mM})$ & $3 \times 10^{-2}$ \\
\hline$t$ & Time & seconds & 0 \\
\hline$g$ & GPVI & molecules & 5000 \\
\hline$s$ & Cytosolic Syk & molecules & 2763 \\
\hline$G$ & GPVI-Ligand Complex & molecules & 0 \\
\hline$G^{p}$ & Phosphorylated Receptor & molecules & 0 \\
\hline$G_{0}^{b}$ & Syk-Receptor Complex & molecules & 0 \\
\hline$G_{1}^{b}$ & Syk with Y525 Phosphorylated & molecules & 0 \\
\hline
\end{tabular}

Table 1. Variables used in the signaling model. Initial concentrations of GPVI and cytosolic Syk were determined with immunoblotting. During experiments, the collagen-related peptide used as the ligand was always maintained at high saturation levels and so a non-rate limiting initial value was selected. ${ }^{1}$ 


\begin{tabular}{|c|c|c|c|}
\hline Parameter & Description & Units & Value \\
\hline$k_{1}$ & Rate of Ligand Binding & $\frac{\mathrm{m}^{3}}{\mathrm{moles} \cdot \mathrm{s}}$ & 8 \\
\hline$k_{-1}$ & Rate of Ligand Dissociation & $\mathrm{s}^{-1}$ & $3.02 \times 10^{-2}$ \\
\hline$V_{e}$ & Extracellular Volume per Cell & $\mathrm{m}^{3}$ & $3.3 \times 10^{-9}$ \\
\hline$V_{p}$ & Platelet Volume & $\mathrm{m}^{3}$ & $7.4 \times 10^{-18}$ \\
\hline$k_{2}$ & Rate of Receptor Phosphorylation & $\mathrm{s}^{-1}$ & $3.02 \times 10^{-2}$ \\
\hline$k_{3}$ & Rate Syk Binds to Receptor & $\mathrm{s}^{-1}$ & $9.55 \times 10^{5}$ \\
\hline$\rho_{1}$ & Rate of Syk Phosphorylation & $\mathrm{s}^{-1}$ & $5.13 \times 10^{-1}$ \\
\hline$\gamma_{1}$ & Rate of Syk Dephosphorylation & $\mathrm{s}^{-1}$ & 3.53 \\
\hline$T$ & Experimental Duration & $\mathrm{s}$ & 250 \\
\hline
\end{tabular}

Table 2. Parameters used in the model Equations $1 \mathrm{a}-1 \mathrm{~g}$, and in their non-dimensionalization Equations $2 \mathrm{a}-2 \mathrm{~g}$. $k_{1}$ and $k_{-1}$, the rate constants for ligand association and dissociation, were previously reported. ${ }^{16}$ The other rate constants were determined through parameter fitting using estimates based on the kinetics of similar proteins or of proteins in different cell lines. Platelet volume was determined during sample collection and the extracellular volume was calculated from there. ${ }^{1}$

In summary, the set of ODEs given by Dunster et al. ${ }^{1}$ to represent the initial steps of platelet activation are below.

$$
\begin{aligned}
\frac{d l}{d t} & =-\frac{k_{1}}{V_{e} A_{v}} g l+\frac{k_{-1}}{V_{e} A_{v}} G \\
\frac{d g}{d t} & =-k_{1} g l+k_{-1} G \\
\frac{d G}{d t} & =k_{1} g l-k_{-1} G-k_{2} G \\
\frac{d G^{p}}{d t} & =k_{2} G-\frac{k_{3}}{V_{p} A_{v}} G^{p} s \\
\frac{d s}{d t} & =-\frac{k_{3}}{V_{p} A_{v}} G^{p} s \\
\frac{d G_{0}^{b}}{d t} & =\frac{k_{3}}{V_{p} A_{v}} G^{p} s-\rho_{1} G_{0}^{b}+\gamma_{1} G_{1}^{b} \\
\frac{d G_{1}^{b}}{d t} & =\rho_{1} G_{0}^{b}-\gamma_{1} G_{1}^{b}
\end{aligned}
$$

Equation 1a.

Equation $1 b$.

Equation 1c.

Equation 1d.

Equation 1e.

Equation 1f.

Equation 1g.

Definitions of all variables and parameters are given in Tables 1 and 2, respectively.

This system of equations can be solved numerically (Figure 2), allowing relative concentrations to be compared visually throughout the signaling cascade. This approach also shows the approximate times at which different stages of the cascade are switched on or off and when steady state is reached. Subsequent decomposition and simplification of this system can be compared to these numerical solutions for validation.

\section{Formulation of Assumptions}

The clotting signal cascade is a complex system with a myriad of enzymes, secondary messengers, and ligands that work to regulate its activation and steady state. This model operates under several assumptions, ${ }^{1}$ and only considers the activation of GPVI and the phosphorylation of Syk. The following assumptions impose spatial uniformity on the system, while granting the ability to describe it via mass action kinetics. Other simplifications made in this model include: no feedback mechanisms, a single phosphorylation site, all or nothing responses, and single pathway responses. This simplifies the underlying biological mechanisms of the clotting cascade, but allows us to obtain explicit solutions for all the reactants in the system. 


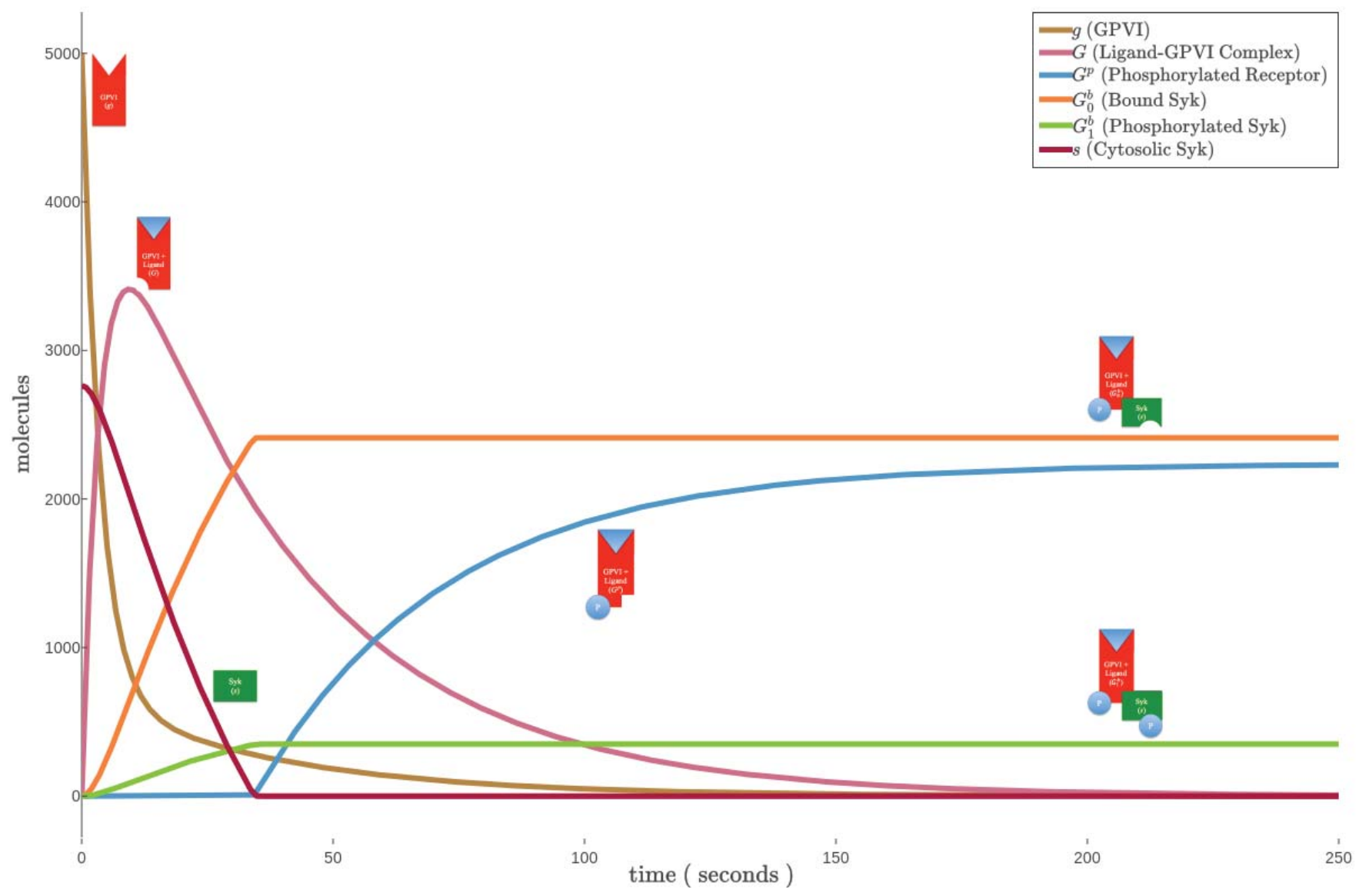

Figure 2. Numerical Solutions to Signaling Cascade. The solutions to the system of ODEs (Equations $1 \mathrm{~b}$ through $1 \mathrm{~g}$ ) with initial conditions and parameters as detailed in Tables 1 and 2. The proteins present during the start of the reaction $(g$ and $s)$ get consumed as the reaction proceeds and go to 0 . The intermediate, $\mathrm{G}$, is initially created, but as the reaction proceeds gets consumed. $G^{p}$ gets created, but its reaction with $s$ is so fast that it is immediately converted into $G_{0}^{b}$ which can reversibly be phophorylated into $G_{1}^{b}$. Once all of $s$ is consumed, no new $G_{0}^{b}$ can be made so $G^{p}$ begins to accumulate and $G_{0}^{b}$ and $G_{1}^{b}$ reach an equilibrium.

Two important assumptions for ODE formulation are:

Assumption 1: The platelet's surface area and receptor density is constant.

Justification: Anuclear cells, such as platelets, lack the requisite genetic material for the production of additional cellular components. This holds for the proteins, phospholipids, or cholesterols that would need to be incorporated into the cellular membrane.

Assumption 2: Cytosolic, membrane, and extracellular components are evenly distributed.

Justification: Rapid diffusion leads to an even dispersal of proteins within the platelet and on its surface. This implies that spatial variation is irrelevant. Further, the platelet's conformational change, induced by the cascade, occurs on a larger timescale than the initial signaling phenomenon of interest.

It is important to note that this model is the most simple model offered by Dunster et al. ${ }^{1}$ It ignores the feedback mechanisms and increased biological fidelity of later models. 


\section{MATHEMATICAL APPROXIMATION}

Non-Dimensionalization

The system of ODEs is non-dimensionalized. In addition to reducing the number of parameters, this provides a means to compare the rates of each reaction step of platelet signaling activation, which is shown in Figure 2. This helps to determine which kinetic steps are relatively fast or slow in comparison to the other steps. Table 3 presents the non-dimensionalized parameters, variables and their definitions in terms of the original, dimensional parameters.

The dimensionless ODEs are as follows.

$$
\begin{aligned}
\frac{d \Gamma}{d \tau} & =-\hat{\alpha}_{1} \varphi \Gamma+\alpha_{-1} \Phi \\
\frac{d \varphi}{d \tau} & =-\hat{\alpha}_{1} \varphi \Gamma+\alpha_{-1} \Phi \\
\frac{d \Phi}{d \tau} & =\hat{\alpha}_{1} \varphi \Gamma-\alpha_{-1} \Phi-\beta \Phi \\
\frac{d \Phi^{p}}{d \tau} & =\beta \Phi-\eta \Phi^{p} \sigma \\
\frac{d \sigma}{d \tau} & =-\eta \Phi^{p} \sigma \\
\frac{d \Phi_{0}^{b}}{d \tau} & =\eta \Phi^{p} \sigma-\epsilon \Phi_{0}^{b}+\theta \Phi_{1}^{b} \\
\frac{d \Phi_{1}^{b}}{d \tau} & =\epsilon \Phi_{0}^{b}-\theta \Phi_{1}^{b}
\end{aligned}
$$

Equation 2a.

Equation $2 b$.

Equation 2c.

Equation 2d.

Equation 2e.

Equation $2 \mathrm{f}$.

Equation 2g.

\begin{tabular}{|c|c|c|}
\hline Variable & Definition & Initial Value \\
\hline$\tau$ & $\frac{t}{T}$ & 0 \\
\hline$\Gamma$ & $\frac{t V_{e} A_{v}}{g_{0}}$ & $1.2 \times 10^{12}$ \\
\hline$\varphi$ & $\frac{g}{g_{0}}$ & 1 \\
\hline$\sigma$ & $\frac{s}{g_{0}}$ & 0.55 \\
\hline$\Phi$ & $\frac{G}{g_{0}}$ & 0 \\
\hline$\Phi^{p}$ & $\frac{G^{p}}{g_{0}}$ & 0 \\
\hline$\Phi_{0}^{b}$ & $\frac{G_{0}^{b}}{g_{0}}$ & 0 \\
\hline$\Phi_{1}^{b}$ & $\frac{G_{1}^{b}}{g_{0}}$ & 0 \\
\hline
\end{tabular}

(a)

\begin{tabular}{|c|c|c|}
\hline Parameter & Definition & Value \\
\hline$\hat{\alpha}_{1}$ & $\frac{k_{1} T g_{0}}{V_{e} A_{v}}$ & $5.03 \times 10^{-9}$ \\
\hline$\alpha_{1}$ & $k_{1} T l_{0}$ & 60 \\
\hline$\alpha_{-1}$ & $k_{-1} T$ & 8 \\
\hline$\beta$ & $k_{2} T$ & 8 \\
\hline$\eta$ & $\frac{k_{3}}{V_{p} A_{v}} T g_{0}$ & $2.68 \times 10^{5}$ \\
\hline$\epsilon$ & $\rho_{1} T$ & 128 \\
\hline$\theta$ & $\gamma_{1} T$ & 882 \\
\hline
\end{tabular}

(b)

Table 3. Components of the Non-Dimensional System of ODEs. These tables represent the dimensionless variables (a) and parameters (b) from Dunster et al. ${ }^{1}$ Their corresponding values can be found in Table 1 and Table 2, respectively.

A similar labeling system to the dimensional equations, Equations $1 \mathrm{a}-1 \mathrm{~g}$, is used to allow for easier comparison of the corresponding equations. The dimensionless equations are presented in the order that the reactions shown in Figure 1 occur. Equations $2 \mathrm{a}-2 \mathrm{~g}$ can also be written as a series of chemical reactions. This helps illustrate the approach taken to solve each step.

$$
\Gamma+\varphi \frac{\hat{\alpha}_{1}}{\hat{\alpha}_{-1}} \Phi \stackrel{\beta}{\longrightarrow} \Phi^{p} \underset{\eta}{\stackrel{\sigma}{\longrightarrow}} \Phi_{0}^{b} \underset{\theta}{\stackrel{\epsilon}{\rightleftharpoons}} \Phi_{1}^{b}
$$

The above shows a cascade reaction with non-dimensionalized variables. The Ligand $(\Gamma)$ binds to GPVI $(\varphi)$ to form the GPVI-Ligand Complex $(\Phi)$. The GPVI-Ligand Complex is subsequently phosphorylated $\left(\Phi^{p}\right)$, allowing Cytosolic Syk $(\sigma)$ 
to join and form the Syk-Receptor Complex $\left(\Phi_{0}^{b}\right)$. The Syk-Receptor complex exists at steady state both with Tyrosine 525 phosphorylated $\left(\Phi_{1}^{b}\right)$ and without $\left(\Phi_{0}^{b}\right)$.

\section{Analytical Solution for Equation 2 a}

Numerical integration of the ODEs shows that the ligand concentration is in a pseudo-steady state. After the platelets are exposed to collagen, the rate of change of the ligand is basically zero for the rest of the cascade, and the concentration of the ligand is constant during the period of interest.

Therefore, instead of Equation 1a, the ligand variable is held constant at its initial condition, $1.2 \times 10^{12} \frac{\mathrm{moles}}{\mathrm{m}^{3}}$.

$$
\Gamma(\tau)=\Gamma_{0}
$$

Equation 3a.

Equation $2 \mathrm{~b}$ and Equation $2 \mathrm{c}$ are modified to account for this, and allow Equation $2 \mathrm{a}$ to be removed from the system of ODEs: $\Gamma$ is combined with $\hat{\alpha}_{1}$ to create the $\alpha_{1}$ parameter, $\alpha_{1}=\hat{\alpha}_{1} \Gamma_{0}$.

Analytical Solutions for Equations $2 b$ and $2 c$

These Equations describe the formation of the GPVI-Ligand complex:

$$
\underset{\text { Ligand }}{\Gamma+\varphi} \frac{\hat{\alpha}_{1}}{\alpha_{-1}} \underset{\begin{array}{c}
\text { GPVI-Ligand } \\
\text { Complex }
\end{array}}{\Phi}
$$

In subsequent reactions, $\Phi$ is consumed with rate $\beta$. With the ligand concentration constant, Equations $2 \mathbf{b}$ and $2 \mathbf{c}$ form a closed system. This allows them to be solved exactly by first converting them to matrix form, $\mathbf{x}^{\prime}=\mathbf{A x}$ :

$$
\left(\begin{array}{c}
\frac{d \varphi}{d \tau} \\
\frac{d \Phi}{d \tau}
\end{array}\right)=\left(\begin{array}{cc}
-\alpha_{1} & \alpha_{-1} \\
\alpha_{1} & -\left(\alpha_{-1}+\beta\right)
\end{array}\right) \times\left(\begin{array}{c}
\varphi \\
\Phi
\end{array}\right)
$$

Equation 4.

Finding the eigenvalues and eigenvectors of the matrix above allows an exact, analytical solution to be found (Supplement A).

It was found that

$$
\begin{aligned}
& \varphi(\tau)=c_{1} e^{\lambda_{1} \tau}+c_{2} e^{\lambda_{2} \tau}, \\
& \Phi(\tau)=c_{3} e^{\lambda_{1} \tau}-c_{3} e^{\lambda_{2} \tau}
\end{aligned}
$$

Equation 3b.

Equation 3c.

where $c_{1}=0.137, c_{2}=0.863, c_{3}=0.9698, \lambda_{1}=-6.6145$, and $\lambda_{2}=-68.4855$.

\section{Analytical Approximations for Equations $2 d$ and $2 e$}

The ODEs for cytosolic Syk and phosphorylated GPVI, produced by a previous reaction, form a coupled system, resulting in the formation of the Syk-Receptor complex:

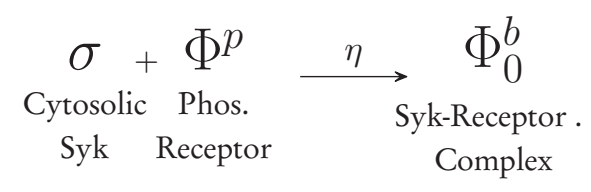




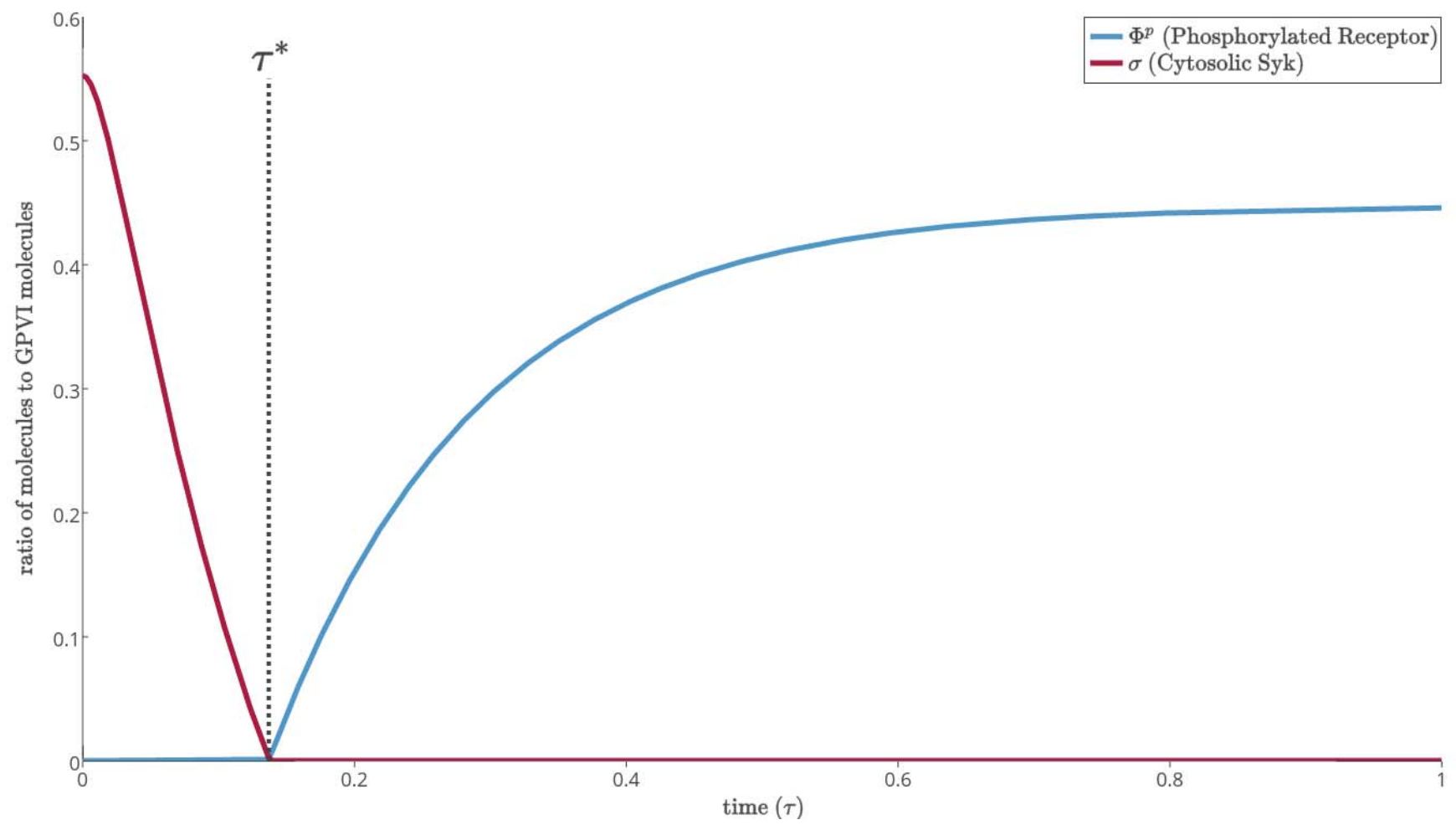

Figure 3. Partitions lending to asymptotic analysis.

The reaction of Syk with phosphorylated GPVI (Equations $\mathbf{2} \mathbf{d}$ and $\mathbf{2 e}$ ) occurs so quickly (with the non-dimensionalized rate constant, $\eta=2.68 \times 10^{5}$ ) that if two reactants are present together, the reaction moves forward. Because of this driving force, phosphorylated GPVI is consumed almost as quickly as it is produced and only begins to accumulate once all of the Syk has reacted. Taking this into account, solutions can be found via asymptotic analysis.

The differential equations can be solved piece-wise with respect to the availability of free $\sigma$. The time at which $\sigma$ is exhausted is labeled $\tau^{*}$ (Figure 3).

Analysis for $\tau<\tau^{*}$

Because $\eta$ is so large, any Syk in solution will consume $\Phi^{p}$ as soon as it is produced. This assumption has implications for both $\frac{d \Phi^{p}}{d \tau}$ (Equation $2 \mathrm{~d}$ ) and $\frac{d \sigma}{d \tau}$ (Equation 2e):

$$
\left(\frac{d \Phi^{p}}{d \tau} \approx 0=\beta \Phi-\eta \Phi^{p} \sigma\right) \Longrightarrow\left(\frac{d \sigma}{d \tau}=-\eta \Phi^{p} \sigma \approx-\beta \Phi\right)
$$

Equation 5.

These assumptions state that changes in $\sigma$ will be proportional to the value of $\Phi(\tau)$, solved previously. Therefore, $\sigma(\tau)$ can be approximated with

$$
\sigma(\tau)=\sigma_{0}-\int_{0}^{\tau} \beta \Phi\left(\tau^{\prime}\right) d \tau^{\prime}, \text { for } 0 \leq \tau<\tau^{*}
$$

Equation 6. 
This gives the following partial approximation for $\sigma(\tau)$ and $\Phi^{p}$ :

$$
\begin{aligned}
\sigma(\tau) & =c_{4} e^{\lambda_{1} \tau}-c_{5} e^{\lambda_{2} \tau}-c_{6}, \\
\Phi^{p} & =0
\end{aligned}
$$

with $\tau \in\left[0, \tau^{*}\right), c_{4}=1.107, c_{5}=0.1069$, and $c_{6}=0.4474$.

Analysis for $\tau \geq \tau^{*}$

As $\tau$ reaches $\tau^{*}, \sigma$ reaches 0 and $\Phi^{p}$ can begin to grow (Figure 3). This allows a similar, but converse, approach to approximating $\Phi^{p}$ and $\sigma$ :

$$
\left(\sigma(\tau) \approx 0, \frac{d \sigma}{d \tau} \approx 0\right) \Longrightarrow \frac{d \Phi^{p}}{d \tau}=\beta \Phi
$$

An approximation to Equation $2 \mathrm{~d}$ can now be found via integration:

$$
\Phi^{p}(\tau)=\int_{\tau^{*}}^{\tau} \beta \Phi\left(\tau^{\prime}\right) d \tau^{\prime}, \text { for } \tau^{*} \leq \tau \leq 1
$$

Equation 8.

With an approximation of $\Phi^{p}$, piecewise solutions for $\sigma$ and $\Phi^{p}$ can be constructed, with $\tau \in[0,1], c_{4}=1.107, c_{5}=0.1069$, and $c_{6}=0.4474$ :

$$
\begin{aligned}
\Phi^{p}(\tau) & = \begin{cases}0 & \text { for } \tau<\tau^{*} \\
-c_{4} e^{\lambda_{1} \tau}+c_{5} e^{\lambda_{2} \tau}+c_{6} & \text { for } \tau \geq \tau^{*}\end{cases} \\
\sigma(\tau) & = \begin{cases}c_{4} e^{\lambda_{1} \tau}-c_{5} e^{\lambda_{2} \tau}-c_{6} & \text { for } \tau<\tau^{*} \\
0 & \text { for } \tau \geq \tau^{*},\end{cases}
\end{aligned}
$$

Equation 3d.

Equation 3e.

The large size of $\eta$ is reflected in the high affinity that cytosolic Syk has for the GPVI-ligand complex. Also, since cytosolic Syk binds quickly and irreversibly to the GPVI complex, it acts as a molecular clock that determines $\tau^{*}$, which is the time when cytosolic Syk is fully consumed and the concentration of phosphorylated GPVI can grow.

From these equations, the intersection was used to determine $\tau^{*}$, determined from:

$$
\left(\lim _{\tau \rightarrow \tau^{*-}} \sigma(\tau)=\right) 0 \Longrightarrow\left(\tau^{*}=0.137\right)
$$

Equation 9.

\section{Analytical Approximation for Equations $2 f$ and $2 g$}

These equations define the concentrations of $\Phi_{0}^{b}$ and $\Phi_{1}^{b}$, and involve the reaction rates $\epsilon$ and $\theta$ :

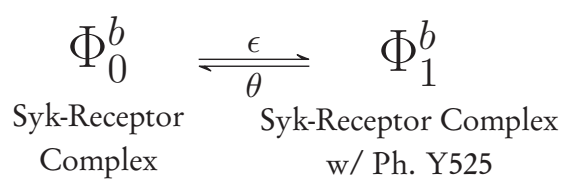

The Syk-Receptor complex is also created at rate $\eta$ as a result of the coupling of cytosolic Syk, $\sigma$ and the phosphorylated receptor, $\Phi^{p}$. We will now solve for $\Phi_{1}^{b}(\tau)$ by eliminating $\Phi_{0}^{b}$ from Equations $2 \mathbf{f}$ and $\mathbf{2 g}$. 
Analysis for $\tau<\tau^{*}$

The previous section established that, for $\tau<\tau^{*}$, we have $\eta \Phi^{p} \sigma \approx \beta \Phi$. This is used in the first step of finding an analytical approximation for $\Phi_{1}^{b}$, solving $\frac{d \Phi_{0}^{b}}{d \tau}$ (Equation $2 \mathbf{f}$ ) for $\Phi_{0}^{b}$ :

$$
\begin{aligned}
\frac{d \Phi_{0}^{b}}{d \tau} & =\beta \Phi-\epsilon \Phi_{0}^{b}+\theta \Phi_{1}^{b} \\
\Phi_{0}^{b} & =\frac{1}{\epsilon}\left(\frac{d \Phi_{1}^{b}}{d \tau}+\theta \Phi_{1}^{b}\right) .
\end{aligned}
$$

Equation $2 \mathrm{~g} *$

After substituting for $\Phi$ from Equation 3c, a solution can be found (see Supplemental C for details):

$$
\Phi_{1}^{b}(\tau)=c_{7}+c_{8} e^{\lambda_{1} \tau}+c_{9} e^{\lambda_{2} \tau}
$$

Equation 10.

with the following constants: $c_{7}=0.126, c_{8}=-0.141, c_{9}=0.0146$.

This solution can be substituted into Equation $2 \mathrm{~g}^{*}$ above, for $\tau<\tau^{*}$. Therefore one has

$$
\Phi_{0}^{b}(\tau)=c_{10} e^{\lambda_{1} \tau}+c_{11} e^{\lambda_{2} \tau}+c_{12}
$$

Equation 11.

with the following $\tau \in\left[0, \tau^{*}\right), c_{10}=-0.9628$, and $c_{11}=0.093, c_{12}=0.867$.

Analysis for $\tau \geq \tau^{*}$

The steady state values for $\Phi_{0}^{b}$ and $\Phi_{1}^{b}$ are well-approximated by their values at $\tau^{*}$ (Supplemental C):

$$
\begin{aligned}
& \Phi_{1}^{b}\left(\tau^{*}\right)=0.069, \\
& \Phi_{0}^{b}\left(\tau^{*}\right)=0.478 .
\end{aligned}
$$

Equation 12a.

Equation 12b.

With these components, solutions can be constructed over the whole domain.

$$
\begin{aligned}
\Phi_{0}^{b}(\tau) & = \begin{cases}c_{10} e^{\lambda_{1} \tau}+c_{11} e^{\lambda_{2} \tau}+c_{12} & \text { if } \tau<\tau^{*} \\
0.478 & \text { if } \tau \geq \tau^{*}\end{cases} \\
\Phi_{1}^{b}(\tau) & = \begin{cases}c_{7}+c_{8} e^{\lambda_{1} \tau}+c_{9} e^{\lambda_{2} \tau} & \text { if } \tau<\tau^{*} \\
0.069 & \text { if } \tau \geq \tau^{*}\end{cases}
\end{aligned}
$$

Equation 3f.

Equation 3g. 


\section{Full Analytical Solutions for Platelet Activation}

For the series of reactions (illustrated in Figure 1) the explicit solutions are

$$
\begin{aligned}
\Gamma(\tau) & =\Gamma_{0}, \\
\varphi(\tau) & =c_{1} e^{\lambda_{1} \tau}+c_{2} e^{\lambda_{2} \tau}, \\
\Phi(\tau) & =c_{3} e^{\lambda_{1} \tau}-c_{3} e^{\lambda_{2} \tau}, \\
\Phi^{p}(\tau) & =\left\{\begin{array}{ll}
0 & \text { for } \tau<\tau^{*} \\
-c_{4} e^{\lambda_{1} \tau}+c_{5} e^{\lambda_{2} \tau}+c_{6} & \text { for } \tau \geq \tau^{*}
\end{array},\right. \\
\sigma(\tau) & =\left\{\begin{array}{ll}
c_{4} e^{\lambda_{1} \tau}-c_{5} e^{\lambda_{2} \tau}-c_{6} & \text { for } \tau<\tau^{*} \\
0 & \text { for } \tau \geq \tau^{*}
\end{array},\right. \\
\Phi_{0}^{b}(\tau) & =\left\{\begin{array}{ll}
c_{10} e^{\lambda_{1} \tau}+c_{11} e^{\lambda_{2} \tau}+c_{12} & \text { for } \tau<\tau^{*} \\
0.478 & \text { for } \tau \geq \tau^{*}
\end{array},\right. \\
\Phi_{1}^{b}(\tau) & = \begin{cases}c_{7}+c_{8} e^{\lambda_{1} \tau}+c_{9} e^{\lambda_{2} \tau} & \text { for } \tau<\tau^{*} \\
0.069 & \text { for } \tau \geq \tau^{*},\end{cases}
\end{aligned}
$$

Equation 14a.

Equation 14b.

Equation 14c.

Equation 14d.

Equation 14e.

Equation 14f.

Equation 14g.

with $\lambda_{1}=-6.6145$, and $\lambda_{2}=-68.4855, c_{1}=0.137, c_{2}=0.863, c_{3}=0.9698, c_{4}=1.107, c_{5}=0.1069, c_{6}=0.4474$, $c_{7}=0.126, c_{8}=-0.141, c_{9}=0.0146, c_{10}=-0.9628, c_{11}=0.093$, and $c_{12}=0.867$, and $\tau^{*}=0.137$.

From Figure 4, the analytic solutions compare favorably to the numerical ones.

\section{CONCLUSIONS}

The system of ODEs from Dunster et al.'s Model A is simplified by first finding its dimensionless equivalent. This process allowed different concentrations and rate kinetics to be directly compared. In addition, the approximate analytical solutions for all the ODEs were found. These solutions are more accessible than the coupled system of differential equations, and each component can now be viewed separately.

Equation 14a shows that the ligand concentration is held constant at its initial value. This is permissible since the right hand side of Equation 2a, the ligand ODE, reaches zero shortly after time zero. It also shows the extent to which phosphorylated Syk functions as a biological clock. When $t$ is equal to approximately 34 seconds, cytosolic Syk is depleted and allows the signal cascade to reach a pseudo steady-state.

The asymptotic, approximate analytic solutions favorably compare to Dunster et al.'s numerical solutions. They maintain its accuracy and scope while providing solutions that are explicit and easier to understand. Further, the underlying biology is more readily exposed; $\tau^{*}$, the effective phosphorylation time scale, marks a clear threshold in the cascade.

The timing mechanism, especially, is shown by the distinct changes in concentrations seen in Figure 4. At $\tau^{*}$, the effective phosphorylation time scale, there is a switch in qualitative behavior for many of the reactants. This is the time that it takes for several of the reactants, bound, phosphorylated, and cytosolic Syk, to reach steady state. These steady state levels then feed into subsequent downstream reactions that are not described in this simplified model. The explicit solutions indicate a coordinated timing mechanism that either activates or deactivates complexes within this cascade.

The experimental data, to which Dunster et al.'s models were parameterized, indicates an overshoot and subsequent undershoot of phosphorylated Syk at $\tau^{*}$. This often arises in systems featuring strong feedback mechanisms. ${ }^{17}$ In fact, Dunster et al. offers models of increasing complexity that more accurately capture this phenomenon. ${ }^{1}$ This is accomplished by introducing more granularity to the phosphorylation of cytosolic Syk on Y525, and an additional phosphorylation site on Y323. 


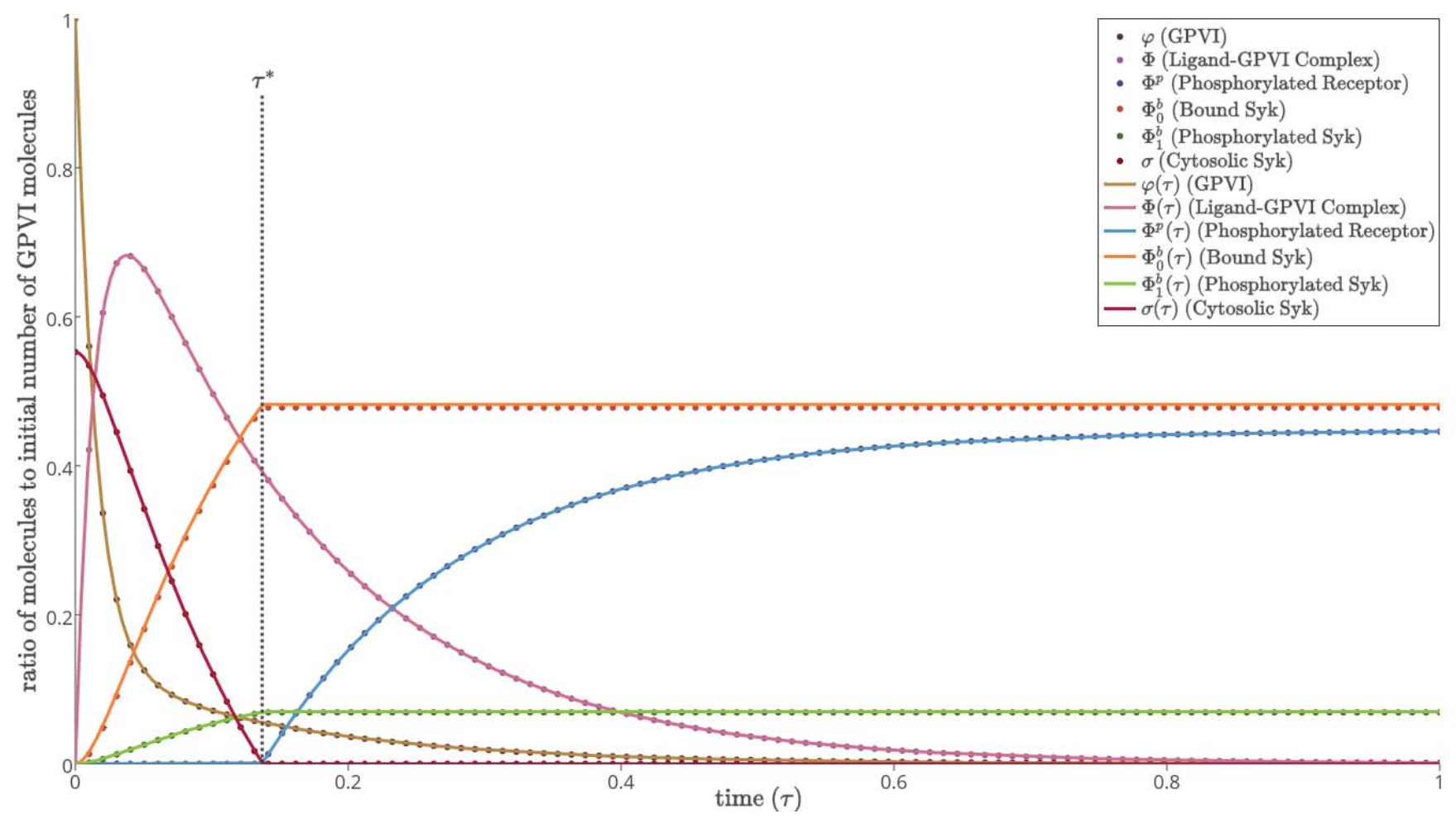

Figure 4. Approximate analytical solutions for the ODEs, $\varphi(\tau), \Phi(\tau), \sigma(\tau), \Phi^{p}(\tau), \Phi_{1}^{b}(\tau)$, and $\Phi_{0}^{b}(\tau)$, with respect to time. The dimensionless versions of Dunster et al.'s ODEs for the GPVI $(\varphi)$, the GPVI complex $(\Phi)$, phosphorylated GPVI ( $\left.\Phi^{p}\right)$, and the cytosolic Syk $(\sigma)$ were solved to create functions dependent on time. The dotted lines represent the numerical solutions from Dunster et al. while the solid lines are the analytical solutions. Cytosolic Syk serves as a molecular clock, marking the timescale of effective phosphorylation. Its depletion at $\tau^{*}$ correlates to a dramatic change in the cascade's dynamics.

However, these models do not include additional steps in the platelet's activation cascade. An interesting extension to this work would be to use our analytic approximations for the ligand, GPVI, and Syk (Equations 14a-14e) as inputs to more complicated models of platelet activation, such as Models B and C of Dunster et al. ${ }^{1}$ 


\section{REFERENCES}

1. Dunster, Joanne L., Mazet, Francoise, Fry, Michael J., Gibbins, Jonathan M., and Tindall, Marcus J. Regulation of Early Steps of GPVI Signal Transduction by Phosphatases: A Systems Biology Approach. PLOS Computational Biology, 11(11):1-26, 2015.

2. Watson, S. P., Auger, J. M., McCarty, O. J. T. and Pearce, A. C. GPVI and integrin AlphaIIbBeta3 signaling in platelets. Journal of Thrombosis and Haemostasis, 3(8): 1752-1762, 2005.

3. Ruggeri, ZM. Platelets in atherothrombosis.Nature Medicine, 8(11): 1227-1234, 2002.

4. Stepanek, O, Draber, P, Drobek, A, Horejsi, V and Brdicka, T. Nonredundant Roles of Src-Family Kinases and Syk in the Initiation of B-Cell Antigen Receptor Signaling. Journal of Immunology,190(4): 1807-1818, 2013.

5. Purvis, Jeremy E., Chatterjee, Manash S., Brass, Lawrence F. and Diamond, Scott L. A molecular signaling model of platelet phosphoinositide and calcium regulation during homeostasis and P2Y1 activation. Blood, 112(10):4069-4079, 2008.

6. Thomas, Dafydd H., Getz, Todd M., Newman, Tiffanny N., Dangelmaier, Carol A., Carpino, Nick, Kunapuli, Satya P., Tsygankov, Alexander Y., and Daniel, James L. A novel histidine tyrosine phosphatase, TULA-2, associates with Syk and negatively regulates GPVI signaling in platelets. Blood, 116(14): 2570-2578, 2010.

7. Dangelmaier, Carol A., Quinter, Patericia G., Jin, Jianguo, Tsygankov, Alexander Y., Kunapuli, Satya P., and Daniel, James L. Rapid ubiquitination of syk forllowing GPVI activation in platelets. Blood, 105(10): 3918-3924, 2005.

8. Senis, Yotis A., Mazharian, Alexandra, and Mori, Jun. Src family kinases: at the forefront of platelet activation. Blood, 124(13) 2013-2024, 2014.

9. Tonks, Nicholas K. Protein tyrosine phosphatases - from housekeeping enzymes to master regulators of signal transduction. FEBS Journal 280(2): 346-378, 2013.

10. Tsang, Emily, Giannetti, Anthony M., Shaw, David, Dinh, Marie, Tse, Joyce K. Y., Gandhi, Shaan, Ho, Hoangdung, Wang, Sandra, Papp, Eva and Bradshaw, J. Michael. Molecular Mechanism of the Syk Activation Switch. Journal of Biological Chemistry, 283(47):32650-32659, 2008.

11. Heemskerk, JWM, Bevers, EM, and Lindhout, T. Platelet activation and blood coagulation. Thrombosis and Haemostasis,88(2): 186-193, 2002.

12. Hognestad, Aina, Michelsen, Annika, Brosstad, Frank, Damås, Jan K, Holm, Torbjørn, Simonsen, Svein, Kjekshus, John K, Aukrust, Pål, and Andreassen, Arne K. Platelet activation in heart transplant recipients. Clinical Transplantation, 18(2): 142-147, 2004.

13. Robinson, SD, Harding, SA, Cummins, P, Din, JN, Sarma, J, Davidson, I, Fox, KAA, Boon, NA, and Newby, DE. Functional interplay between platelet activation and endothelial dysfunction in patients with coronary heart disease. Platelets 17(3): 158-162, 2006.

14. Kreis, T. and Vale, R. Guidebook to the Extracellular Matrix, Anchor, and Adhesion Proteins. A Sambrook \& Tooze publication at Oxford University Press.Oxford University Press, 1999.

15. Nieswandt, B., Pleines, I., and Bender, M. Platelet adhesion and activation mechanisms in arterial thrombosis and ischaemic stroke. Journal of Thrombosis and Haemostasis, 9:92-104, 2011.

16. Miura, Y., Takahashi, T., Jung, S., Moroi, M. Analysis of the Interaction of Platelet Collagen Receptor Glycoprotein VI (GPVI) with Collagen. Journal of Biological Chemistry, 277:46197-46204, 2002.

17. Alon, U. Network motifs: Theory and experimental approaches. Nature Revierws.Genetics. 8(6):450-61, 2007. doi: http://dx.doi.org.udel.idm.oclc.org/10.1038/nrg2102. 


\section{SUPPLEMENT A: ANALYTICAL SOLUTIONS FOR $\phi$ AND $\Phi$}

The equations for $\phi$ and $\Phi$ can be solved as an independent system, separate from the other ODEs.

$$
\begin{aligned}
& \frac{d \varphi}{d \tau}=-\alpha_{1} \varphi+\alpha_{-1} \Phi \\
& \frac{d \Phi}{d \tau}=\alpha_{1} \varphi-\alpha_{-1} \Phi-\beta \Phi
\end{aligned}
$$

Equation 15a.

Equation 15b.

In matrix form, $\mathbf{x}^{\prime}=\mathbf{A x}$ :

$$
\left(\begin{array}{c}
\frac{d \varphi}{d \tau} \\
\frac{d \Phi}{d \tau}
\end{array}\right)=\left(\begin{array}{cc}
-\alpha_{1} & \alpha_{-1} \\
\alpha_{1} & -\left(\alpha_{-1}+\beta\right)
\end{array}\right) \times\left(\begin{array}{c}
\varphi \\
\Phi
\end{array}\right) .
$$

Equation 16.

Solving for the eigenvalues of A yields the approximate solutions: $\lambda_{1}=-6.6145, \lambda_{2}=-68.4855$, with corresponding eigenvectors:

$$
\mathbf{v}_{\mathbf{1}}=\left(\begin{array}{c}
0.14003 \\
0.99015
\end{array}\right), \mathbf{v}_{\mathbf{2}}=\left(\begin{array}{c}
-0.66473 \\
0.74709
\end{array}\right)
$$

Equation 17.

Therefore, solutions have the form:

$$
\left(\begin{array}{l}
\varphi \\
\Phi
\end{array}\right)=d_{1} \mathbf{v}_{\mathbf{1}} e^{\lambda_{1} \tau}+d_{2} \mathbf{v}_{\mathbf{2}} e^{\lambda_{2} \tau}
$$

Equation 18.

$d_{1}$ and $d_{2}$ can be found by solving at the initial conditions $\varphi(0)=1$ and $\Phi(0)=0$.

$$
\begin{aligned}
1 & \approx 0.14003 d_{1}-0.66473 d_{2} \\
0 & \approx 0.99015 d_{1}+0.74709 d_{2} \\
d_{1} & \approx 0.97941 \\
d_{2} & \approx-1.29806
\end{aligned}
$$

Therefore, the solutions for $\varphi$ and $\Phi$ are.

$$
\begin{aligned}
& \varphi \approx 0.13715 e^{-6.6145 \tau}+0.86285 e^{-68.4854 \tau} \\
& \Phi \approx 0.96976 e^{-6.6145 \tau}-0.96976 e^{-68.4854 \tau} .
\end{aligned}
$$

\section{SUPPLEMENT B: ANALYTICAL SOLUTIONS FOR $\Phi^{P}$ AND $\sigma$}

Approximate analytical solutions can be found for $\Phi^{p}$ and $\sigma$ via asymptotic analysis.

Because $\eta$ is so large, any Syk in solution will consume $\Phi^{p}$ as soon as it is produced. $\tau^{*}$ denotes the time when $\sigma$ reaches zero and $\Phi^{p}$ can begin to grow. This allows $\Phi^{p}$ and $\sigma$ to be defined piece-wise with respect to $\tau^{*}$ and allow approximate solutions to be obtained.

$$
\begin{gathered}
\sigma(\tau)= \begin{cases}\sigma_{0}-\int_{0}^{\tau} \beta \Phi\left(\tau^{\prime}\right) d \tau^{\prime} & \text { if } 0 \leq \tau<\tau^{*} \\
0 & \text { if } \tau^{*} \leq \tau \leq 1\end{cases} \\
\Phi^{p}(\tau)= \begin{cases}0 & \text { if } 0 \leq \tau<\tau^{*} \\
\int_{\tau^{*}}^{\tau} \beta \Phi\left(\tau^{\prime}\right) d \tau^{\prime} & \text { if } \tau^{*} \leq \tau \leq 1\end{cases}
\end{gathered}
$$

Equation 19.

Equation 20.

The value of $\tau^{*}$ can be found by solving $\sigma\left(\tau^{*}\right)=0$.

$$
\begin{aligned}
0 & =\sigma_{0}-\int_{0}^{\tau^{*}} \beta \Phi\left(\tau^{\prime}\right) d \tau^{\prime} \\
& =0.5526-\int_{0}^{\tau^{*}} 7.55\left(0.9698 e^{-6.6145 \tau^{\prime}}-0.9698 e^{-68.4855 \tau}\right) d \tau^{\prime} \\
\Longrightarrow-0.4474 & =-1.107 e^{-6.6145 \tau^{*}}+0.1069 e^{-68.4855 \tau^{*}} \\
\Longrightarrow \tau^{*} & =0.137
\end{aligned}
$$


a particular solution to $\Phi_{1}^{b}$ can be assumed to take the form:

$$
\Phi_{1}^{b}(\tau)=A e^{-6.6145 \tau}+B e^{-68.4855 \tau} .
$$

Equation 22.

Substituting into Equation A2 and comparing coefficients resulted in the following solutions of $A$ and $B$ :

$$
\begin{aligned}
& A=-0.141 \\
& B=0.0146
\end{aligned}
$$

Equation 23.

Equation 24.

Now, the solution to the differential equation is a combination of the homogeneous solution and the particular solution.

$$
\Phi_{1}^{b}=f_{1}+f_{2} e^{-1010.75 \tau}-0.141 e^{-6.6145 \tau}+0.0146 e^{-68.4855 \tau}
$$

Equation 25.

Solutions for the constants are found by taking the derivative and using the initial values of $\Phi_{1}^{b}(\tau)$ and $\frac{d \Phi_{1}^{b}}{d \tau}$.

$$
\begin{aligned}
& f_{1}=0.126 \\
& f_{2}=6.629 \times 10^{-5}
\end{aligned}
$$

Equation 26.

Equation 27.

The final solution is:

$$
\Phi_{1}^{b}(\tau)=0.126-6.629 \times 10^{-5} e^{-1010.25 \tau}-0.141 e^{-6.6145 \tau}+0.0146 e^{-68.4855 \tau}
$$

Equation 28.

The above solution is only accurate when $0 \leq \tau<\tau^{*}$. To determine the steady state value, the equation must be evaluated at $\tau=\tau^{*}$, where $\tau^{*}=0.137$.

$$
\begin{aligned}
\Phi_{1}^{b}\left(\tau^{*}\right) & =0.126-6.629 \times 10^{-} 5 e^{-1010.25 \tau^{*}}-0.141 e^{-6.6145 \tau^{*}}+0.0146 e^{-68.4855 \tau^{*}} \\
& =0.069
\end{aligned}
$$

Equation 29.

Equation 30.

Equation 31.

The final equation for $\Phi_{1}^{b}(\tau)$ is as follows.

$$
\Phi_{1}^{b}(\tau)= \begin{cases}0.126-6.629 \times 10^{-5} e^{-1010.75 \tau}-0.141 e^{-6.6145 \tau}+0.0146 e^{-68.4855 \tau} & \text { if } 0 \leq \tau<\tau^{*} \\ 0.069 & \text { if } \tau^{*} \leq \tau \leq 1\end{cases}
$$

Equation 32.

Next, an analytical approximation for $\Phi_{0}^{b}$ is determined from the original equation, Equation $2 \mathrm{~g}$. Since $\Phi_{1}^{b}$, is now known, $\Phi_{0}^{b}$ can be solved by substituting the solution for $\Phi_{1}^{b}$ into Equation $2 \mathrm{~g}^{*}$.

$$
\Phi_{0}^{b}=\frac{1}{\epsilon}\left(\frac{d}{d \tau} \Phi_{1}^{b}+\theta \Phi_{1}^{b}\right)
$$

Equation $2 \mathrm{~g} *$

Solving for $\Phi_{0}^{b}$ when $\tau^{*}=0.137$

$$
\begin{aligned}
\Phi_{0}^{b}(0.137) & =-0.9628 e^{-0.9062}+0.093 e^{-9.383}+6.629 \times 10^{-5} e^{-138.49}+0.00272 e^{-17.57}+0.867 \\
\Phi_{0}^{b}\left(\tau^{*}\right) & =0.478
\end{aligned}
$$

Equation 33.

Equation 34.

The final equation comes out to be:

$$
\Phi_{0}^{b}(\tau)= \begin{cases}-0.9628 e^{-6.6145 \tau}+0.093 e^{-68.4855 \tau}+6.629 \times 10^{-5} e^{-1010.75 \tau}+0.00272 e^{-128.25 \tau}+0.867 & \\ 0.478 & \text { if } 0 \leq \tau<\tau^{*} \\ & \text { if } \tau^{*} \leq \tau \leq 1\end{cases}
$$

Equation 35. 
Note: Both $\Phi_{0}^{b}$ and $\Phi_{1}^{b}$ include an $e^{-1010.75 \tau}$ term that does not contribute significantly to the solution. Removing this term from both equations simplifies them to:

$$
\begin{aligned}
& \Phi_{1}^{b}(\tau)= \begin{cases}0.126-0.141 e^{-6.6145 \tau}+0.0146 e^{-68.4855 \tau} & \text { if } \tau \leq \tau^{*} \\
0.069 & \text { if } \tau>\tau^{*}\end{cases} \\
& \Phi_{0}^{b}(\tau)=\left\{\begin{array}{ll}
-0.9628 e^{-6.6145 \tau}+0.093 e^{-68.4855 \tau}+0.00272 e^{-128.25 \tau}+0.867 & \text { if } 0 \leq \tau<\tau^{*} \\
0.478 & \text { if } \tau^{*} \leq \tau \leq 1
\end{array} \quad \text { Equation } 37 .\right.
\end{aligned}
$$

\section{ABOUT THE STUDENT AUTHORS}

Rachel Austin recently graduated from the University of Delaware with a Bachelor of Science in Quantitative Biology and a minor in Dance and is currently serving as an AmeriCorps member with City Year Philadelphia.

Scott Fones is currently a senior at the University of Delaware, pursuing a Bachelor of Science in Quantitative Biology and minoring in Computer Science.

Dominic Santoleri graduated from the University of Delaware in 2017 with Bachelors of Science in Biochemistry and in Quantitative Biology. He is currently pursuing a PhD in Biochemistry and Molecular Biophysics at the University of Pennsylvania.

Kaitlyn Thomesen recently graduated from the University of Delaware with a Bachelor of Science in Quantitative Biology and Economics and a minor in Chemistry and Biochemistry. She is currently pursuing her Master of Business Administration with a concentration in Healthcare Management at the University of Delaware.

\section{PRESS SUMMARY}

The ordinary differential equations proposed in Model A of Regulation of Early Steps of GPVI Signal Transduction by Phosphatase: A Systems Biology Approach by JL Dunster were simplified through the process of non-dimensionalization. The approximated analytical solutions were found and compared against the numerical solutions. With this simplification, a cross comparison for activation kinetics in this phenomenon was performed, allowing for a better understanding of the signal regulation pathway specifically, thereby exposing an effective phosphorylation time scale of about 34 seconds. 